\title{
Estudo da secagem de materiais por meio de Speckle dinâmico
}

\section{RESUM 0}

Sidney Leal da Silva fatec.professor.sidney@gmail.com Brasil.

Daniel José Toffoli Fatec Itaquera, São Paulo, São Paulo, Brasil.

Augusto Cesar Santos augustocesar.santos98@gmail.com Fatec Itaquera, São Pau lo, São Paulo, Brasil.

Henrique Trajano de Araujo ricky trajy@hotmaill.com Fatec Itaquera, São Paulo, São Paulo, Brasil.
Neste trabalho, utilizou-se Speckle dinâmico para verificar o tempo médio de secagem das amostras corretivo líquido, cola de madeira e cola branca. Os padrões de interferência foram obtidos por reflexão e capturados em uma câmera digital após o laser interagir com as superfícies das amostras. Com 0 método THSP obtiveram-se os coeficientes de correlação e por análises gráficas encontrou-se os tempos médios de secagens das amostras: $(3,76 \pm 0,34)$ min para o corretivo líquido, $(26,02 \pm 0,44)$ min para a cola de madeira e $(37,36 \pm 0,33)$ min para a cola tenaz branca. Esses resultados apresentaram maior precisão se comparados aos valores obtidos por método do momento de inércia em THSP, encontrados na literatura, mostrando que o método computacional de correlação é mais adequado para a obtenção dos tempos de secagem para o conjunto de amostras deste trabalho.

PALAVRAS-CHAVE: Interferometria Speckle; CorrelaçãoSpeckle; THSP. 


\section{INTRODUÇÃO}

O Speckle, utilizado nas mais diversas áreas do conhecimento, como ciências agrárias, engenharia e medicina, continua sendo uma técnica que possibilita novas e importantes aplicações tecnológicas, mesmo tendo-se passado séculos desde sua primeira descrição, por Isaac Newton, em seu tratado Optiks de 1730 (NEWTON e ASSIS, 2015).

Uma reflexão difusa ocorre quando a luz visível incide em uma superfície rugosa ou dinâmica, o que por sua vez gera um padrão de interferências que possui distribuição aleatória estática ou dinâmica de intensidades luminosas, sendo muitas vezes percebido a olho nu (dependendo das condições do experimento) ou ainda podendo ser capturado por uma câmera. Este padrão de interferências se assemelha à imagem de um granulado ou fervilhado óptico e foi inicialmente percebido por Newton e posteriormente estudado por Exner em 1877, quando notou a estrutura fibrosa formada pela incidência de luz em uma placa de vidro em que estava condensado o vapor de sua respiração. Sua formalizaçãomatemática foi efetuada por von Loue e lord Rayleigh na primeira metade do século XX (ARIZAGA, TRIVI e RABAL, 1999).

Atualmente, esta técnica é bastante difundida e utilizada no campo das ciências agrárias, onde recebe o nome de biospeckle, por utilizar o fenômeno do speckle para a investigação de amostras biológicas, que são dinâmicas em sua natureza e que, portanto, pode provocar reflexão difusa do laser incidente, condição necessária para formação das interferências que serão posteriormente analisadas (M IRA, 2016).

No campo das ciências biológicas o speckle vem atraindo cada vez mais atenção por ser uma técnica não destrutiva, em que não há contato físico com a amostra e por permitir grande precisão nos resultados, visto que deslocamentos da ordem de nanometros (mesma ordem que o comprimento de onda da luz visível) podem ser mensurados (ALVES, 2012).

No campo da medicina a técnica de biospeckle é utilizada, de acordo com Hotta e Vieira (2015), para diagnósticos clínicos variados. 0 speckle tem também apresenta grande valia em áreas como engenharia e física por permitir a determinação de propriedades de materiais (como módulo de elasticidade e coeficiente de Poisson) sem destruir as amostras em estudo (DA SILVA, 2017).

Ainda no campo da engenharia, para citar mais exemplos, Marchiori e Souza (2013) aplicaram o speckle para analisar a rugosidade de uma superfície usinada, e Kuramoto (2011) realizou medidas de vibração através da análise do granulado óptico. Além desta vasta gama de aplicações, o Speckle se destaca pela facilidade de montagem de sua configuração experimental e pela rápida análise dos dados; os resultados podem ser obtidos de maneira sistemática e com grande precisão por meio de modelagem computacional, como mostra o trabalho de da Silva (2017).

A técnica de speckle se utiliza de variados métodos de análise de resultados, que podem ser basicamente divididos em métodos de análise numérica e métodos de rotinas gráficas. Entre os primeiros métodos encontram-se a análise por momento de inércia, a diferença de valores absolutos e a correlação; entre os 
métodos de rotinas gráficas temos o método de Fujii, diferenças generalizadas e 0 método de desvio-padrão. Além destes, temos a história temporal do padrão de Speckle (THSP) e a matriz de ocorrência (MOC), que podem ser classificados como um misto de método numérico e método gráfico (M OREIRA, 2014).

Com uma diversidade de métodos associados a técnica de Speckle, não fica claro para os pesquisadores qual método é mais interessante para cada tipo de análise que será realizada. Dentro deste contexto, o propósito deste trabalho é comparar o uso de dois dos métodos de análise numérica do padrão de interferências Speckle (momento de inércia e correlação) na determinação do tempo de secagem de três amostras (corretivo líquido, cola de madeira e cola branca). Neste trabalho, apresenta-se os resultados obtidos para os tempos de secagem utilizando-se o método da correlação; tais resultados são posteriormente comparados com o trabalho de da Silva et al. (2016), que apresenta um estudo dos tempos de secagem dos mesmos materiais nas mesmas condições, porém utilizando o método de momento de inércia. Através da comparação, pretende-se mostrar qual dentre os dois métodos é o mais indicado para ser utilizado na determinação dos tempos de secagem de materiais.

TEORIA

\section{SPECKLE E HISTÓRIA TEM PORAL DO PADRÃO DE INTENSIDADES}

Speckle é um ramo da óptica que avalia o comportamento estático ou dinâmico do fenômeno de interferência em superfícies iluminadas por uma onda de luz coerente ao longo do tempo, segundo o trabalho de da Silva et al. (2017).

A Figura 1 mostra em tons de cinza a interferência formada durante a iluminação de uma gota de corretivo líquido, liquid paper, por um laser de HélioNeônio (He-Ne). A imagem observadacria um efeito granulado, comumente conhecido como Speckle (DA SILVA, 2016).

Figura 1 - Padrão instantâneo do Speckle de uma gota de corretivo líquido iluminada por um laser de He-Ne

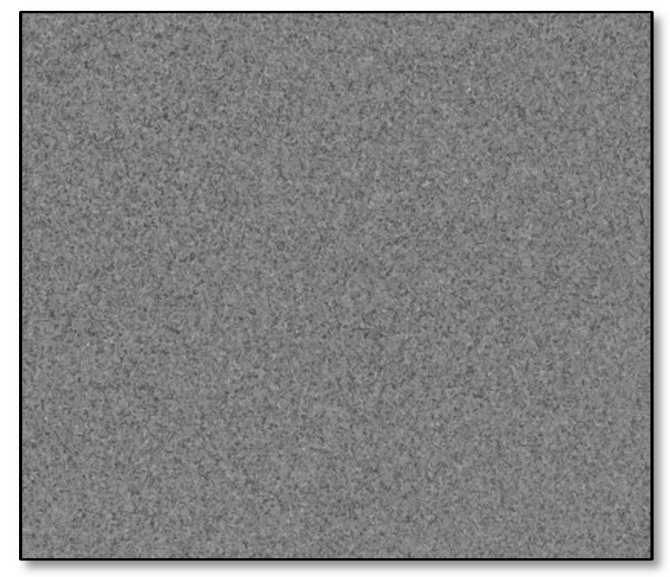

Fonte: DA SILVA (2016) 
Uma boa maneira de compreender a evolução temporal do padrão de interferências produzido pelo speckle dinâmico é utilizar o método Time History Speckle Pattern, THSP, que consiste em registrar computacionalmente a evolução no tempo de um padrão de intensidades em um vídeo, que produz uma sequência de imagens bidimensionais, por meio da coleta de linhas de pixel de cada imagem, em instantes sucessivos, e organizá-las lado a lado em uma imagem intermediária, como mostra os trabalhos de da Silva (2017) e Silva e Muramatsu (2007).

\section{MÉTODO DA CORRELAÇÃO SPECKLE}

A análise de correlação é uma maneira de comparar as intensidades em pontos ou instantes distintos de uma imagem THSP com o objetivo de avaliar a evolução do speckle no espaço ou no tempo, segundo o trabalho de Costa et al (2010). 0 grau de similaridade de uma função temporal f(t) com ela mesma em outro instante arbitrário, $\mathrm{t}+\delta \mathrm{t}$, pode ser analisado pela função de autocorrelação $\gamma(\delta \mathrm{t})$, por transformada de Fourier (ARFKEN e WEBER, 2005; BUTKOV, 1988), tal que

$$
\gamma(\delta \mathrm{t})=\int_{-\infty}^{\infty} \mathrm{f}(\mathrm{t}+\delta \mathrm{t}) \mathrm{f}^{*}(\mathrm{t}) \mathrm{dt}(\mathrm{l})
$$

No entanto, o que um arquivo THSP fornece é um conjunto de intensidades, I(t), espacialmente distribuídas em instantes sucessivos. Então, o conveniente é definir a função de autocorrelação em termos de valores de intensidade.

Assim, por estatística temporal de segunda ordem, que fornece informações acerca da distribuição de velocidades dos espalhadores móveis e estáticos (COSTA et al., 2010; VANIN, GOUFFON e HELENE, 1991), a função $\gamma(\delta \mathrm{t})$, normalizada, pode ser reescrita como

$$
\gamma(\delta \mathrm{t})=\frac{\langle\mathrm{I}(\mathrm{t}) \cdot \mathrm{I}(\mathrm{t}+\delta \mathrm{t})\rangle_{x y}}{\langle\mathrm{I}(\mathrm{t})\rangle_{x y}^{2}}
$$

Na prática, um coeficiente de correlação, $\mu\left(t_{0}, t\right)$, que corresponde a uma medida do grau de semelhança entre o padrão speckle consigo mesmo em um instante ou região diferente, é definido segundo Costa et al. (2010) como

$$
\mu\left(\mathrm{t}_{0}, \mathrm{t}\right)=\frac{\sum_{\mathrm{i}=1}^{\mathrm{N}}\left[\mathrm{I}_{\left(\mathrm{t}_{0}, \mathrm{i}\right)}-\left\langle\mathrm{I}_{\left(\mathrm{t}_{0}, \mathrm{i}\right)}\right\rangle\right]\left[\mathrm{I}_{(\mathrm{t}, \mathrm{i})}-\left\langle\mathrm{I}_{(\mathrm{t}, \mathrm{i})}\right\rangle\right]}{\sqrt{\sum_{\mathrm{i}=1}^{\mathrm{N}}\left[\mathrm{I}_{\left(\mathrm{t}_{0}, \mathrm{i}\right)}-\left\langle\mathrm{I}_{\left(\mathrm{t}_{0}, \mathrm{i}\right)}\right)\right]^{2} \sum_{\mathrm{i}=1}^{\mathrm{N}}\left[\mathrm{I}_{(\mathrm{t}, \mathrm{i})}-\left\langle\mathrm{I}_{(\mathrm{t}, \mathrm{i})}\right)\right]^{2}}}
$$

onde $\mathrm{N}$ corresponde ao total de valores de intensidades e t aos instantes de amostragem. Um baixo nível de atividade tende a apresentar poucasvariações no padrão espalhado ao longo do tempo, ou variações mais lentas, e níveis mais ativos apresentam grandes variações de intensidades no speckle resultante, 0 que implica a queda mais acentuada do coeficiente de correlação, que funciona, então, como um indicador de atividade da amostra. 


\section{MATERIAIS, TÉCNICA E MÉTODO}

\section{AMOSTRAS}

As amostras utilizadas foram: corretivo líquido, cola de madeira e cola branca. Todas as amostras, sem alteração das composiçõesoriginais, foram posicionadas em pedaços quadrados de papel cartão de $2 \mathrm{~cm}$ de lado, em uma área circular com aproximadamente $1 \mathrm{~cm}$ de diâmetro. Para posicioná-las utilizou-se de um conta gotas de $10 \mathrm{ml}$ para cada amostra, no sentido de evitar misturas.

A quantidade variou de amostra para amostra, levando em consideração 0 tempo de secagem. Para o corretivo líquido, foi utilizado um quarto do volume do conta gotas (aproximadamente $2,5 \mathrm{ml}$ ) e, para as colas de madeira e branca, foram utilizados um vigésimo do volume do conta gotas (aproximadamente 0,5 $\mathrm{ml}$ ). As medições foram realizadas na faixa de temperatura entre 20 a $30^{\circ} \mathrm{C}$, à pressão atmosférica (aproximadamente $1 \mathrm{~atm}$ ), e umidade relativa do ar de $40 \mathrm{a}$ $60 \%$.

Vale ressaltar que a coleta de dados (captura do video contendo o padrão de interferência Speckle após iluminação das amostras por laser) foi feita uma única vez, de acordo com a metodologia utilizada no trabalho de da Silva et al (2016). Porém, enquanto o trabalho de 2016 utilizou o método de Momento de Inércia (M I) para análise do padrão de interferência, utiliza-se no presente trabalho o método de correlação.

\section{TÉCNICA DE SPECKLE POR REFLEXÃO}

Na análise, foram capturados vídeos com 4096 quadros em 10 grupos para cada tipo de amostra preparada para a coleta de dados. Os vídeos continham os padrões de interferências dinâmicos que foram gerados pela configuração experimental de speckle por reflexão, esquematizada na Figura 2.

Figura 2 - Esquema da configuração experimental de Speckle por reflexão.

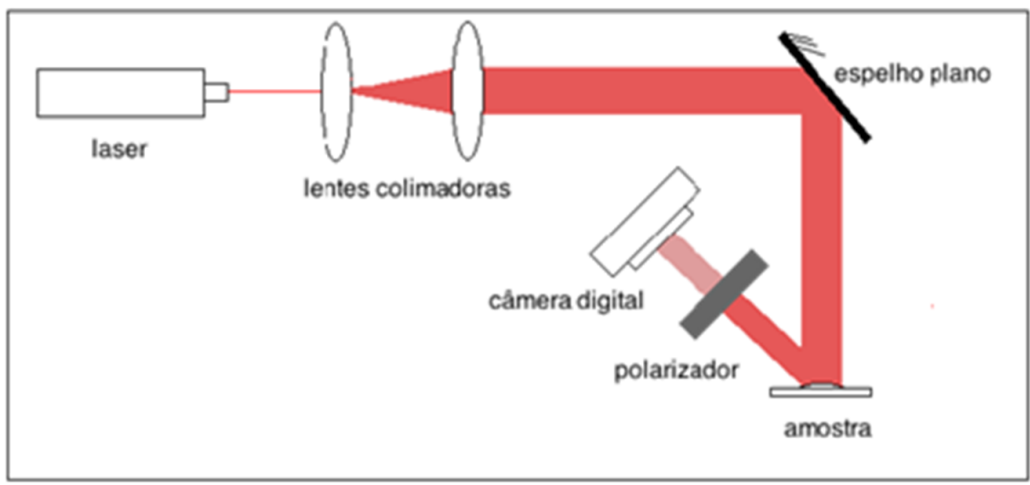

Fonte: DA SILVA et al., 2016 
A fonte de luz foi de um laser com comprimento de onda $\lambda=632,8 \mathrm{~nm}$ (faixa do vermelho) e potência de $5 \mathrm{~mW}$. Posteriormente ao laser, duas lentes convergentes fizeram com que a luz seguisse como uma onda plana de intensidade aproximadamente constante até atingir um espelho plano, que a direcionou até a amostra, produzindo reflexões difusas e um padrão de interferências (HECHT, 2002), formando o granulado estático ou dinâmico do Speckle.

Esse padrão, após um acerto de contraste por meio de um polarizador, foi capturado por uma câmera digital e registrado em arquivo de imagem por um computador.

O processo de captura de dados, em cada amostra, de cada grupo, foi organizado da seguinte forma:

(i) registrou-se um vídeo com $\mathrm{N}$ quadros da amostra seca (obtenção do padrão estático);

(ii) registraram-se vídeos com $\mathrm{N}$ quadros da amostra ao ser retirada do frasco (obtenção do speckle dinâmico);

(iii) utilizaram-se tais vídeos como dados para a determinação dos tempos desejados.

\section{MÉTODO DE ANÁLISE DE DADOS}

A análise foi feita por programas computacionais confeccionados pelo Grupo de Óptica e Aplicações da Fatec Itaquera e seguiram as seguintes etapas:

(i) separou-se os vídeos em quadros, que foram armazenados em arquivos de imagens;

(ii) criou-se 0 arquivo de imagem THSP, a partir dos quadros separados no item anterior, recortando uma linha da mesma posição horizontal de cada imagem e concatenando cada linha em posições verticais consecutivas de uma única imagem;

(iii) determinou-se os coeficientes de correlação $\mu\left(t_{0}, t\right)$, pela Expressão (3), utilizando a relação entre as intensidades médias das colunas de pixels do arquivo de imagem THSP. 0 tempo associado a cada coeficiente de correlação está relacionado à duração de cada coluna de pixels. Assim, por exemplo, se cada coluna tem a duração de t segundos, então, cada $\mu_{\mathrm{i}}$ terá $\mathrm{t}_{\mathrm{i}}=\mathrm{i} * \mathrm{t}$ segundos, com $\mathrm{i} \in[1, \mathrm{n}]$;

(iv) com as relações $\mu$ versus $t$, construiu-se gráficos da amostra base (padrão estático de speckle) sobrepostos aos gráficos das amostras temporais (padrão dinâmico de speckle). Através de um ajuste de curva, pelo método dos mínimos quadrados, encontraram-se as funções $\mu(\mathrm{t})$. Com essas funções, estimaram-se os tempos para a estabilização de cada amostra no limite quando $\mu(\mathrm{t})$ tende a $\mu 0$ obtido com a amostra seca;

(v) calculou-se as médias dos tempos de todas as amostras. 


\section{RESULTADOS E DISCUSSÕES}

Durante o processo de captura, foram criados arquivos de vídeo com 8 bits, na escala de cinza, com 4096 quadros em uma taxa de coleta de 30 quadros por segundo. Esses arquivos armazenaram os padrões estático e dinâmico de speckle para todas as amostras, em todos os grupos: corretivo líquido, cola de madeira e cola branca. 0 padrão estático foi obtido com as amostras secas, respeitando os seus tempos nominais, com exceção do corretivo líquido, já que não foi encontrado valor em especificações do produto.

Cada um dos arquivos foi separado em 16 grupos de 256 quadros e, a partir da taxa de coleta, determinou-se o tempo de duração de cada grupo. Por meio de um programa computacional confeccionado pelo Grupo de Óptica e Aplicações da Fatec Itaquera, cada grupo gerou um THSP, segundo a teoria apresentada. Com os THSP's, foram calculados os coeficientes de correlação. $\mathrm{Na}$ situação estática, os valores médios de coeficientes de correlação são apresentados da Tabela 1.

Tabela 1: Valores médios de correlação para amostras secas

\begin{tabular}{c|c|c|c}
\hline Material & Corretivo líquido & Cola de madeira & Cola tenaz branca \\
\hline Linha base $\left(10^{-1} \mathrm{~s}\right)$ & $1,67 \pm 0,09$ & $1,67 \pm 0,11$ & $2,00 \pm 0,13$ \\
\hline
\end{tabular}

Fonte: Grupo de Óptica e Aplicações da Fatec Itaquera.

Os valores da Tabela 1 representam médias calculadas a partir de 10 medidas efetuadas no processo experimental. Assim, as incertezas estão fundamentadas na Teoria de Erros. Essas médias foram utilizadas como referênciana determinação dos tempos de secagem. As Figuras 3, 4 e 5 apresentam os gráficos médios de correlação em função dos tempos de secagem para as amostras utilizadas nesse trabalho. As linhas azuis representam as linhas de base citadas.

Figura 3 - Gráficos experimentais de correlação versus tempo para o corretivo líquido.

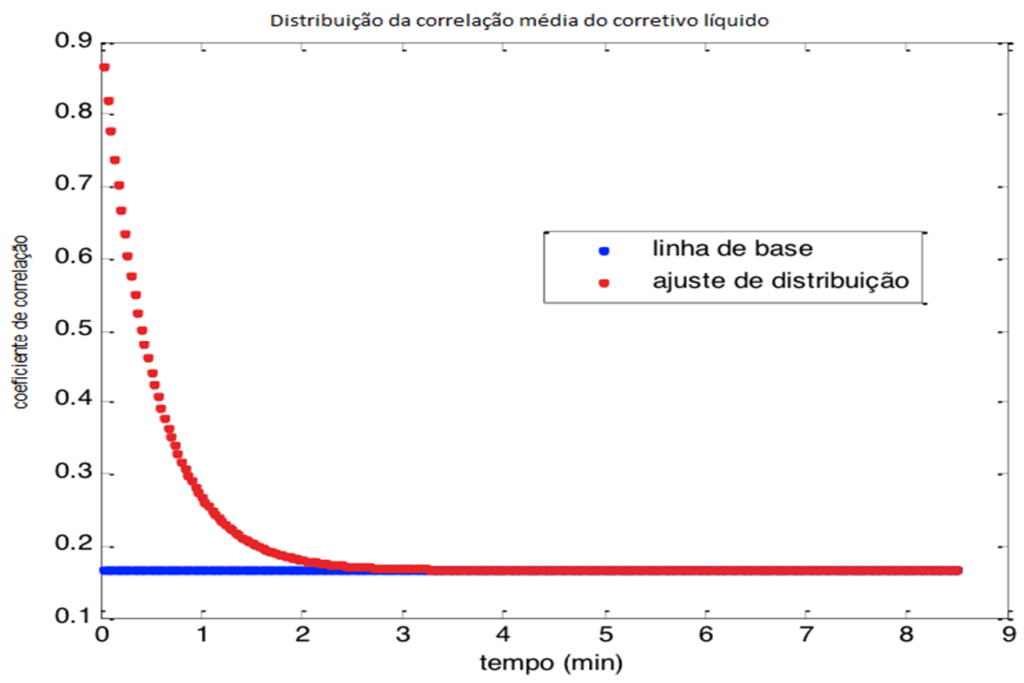

Fonte: Grupo de Óptica e Aplicações da Fatec Itaquera. 
Figura 4 - Gráficos experimentais de correlação versus tempo para a cola de madeira.

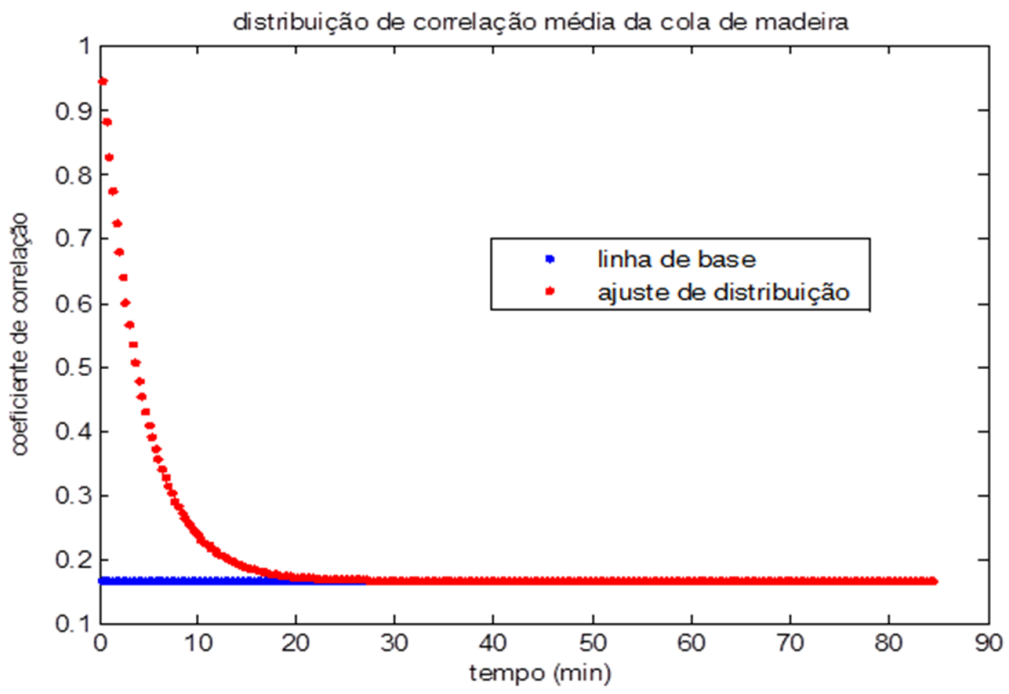

Fonte: Grupo de Óptica e Aplicações da Fatec Itaquera.

Analisando as Figuras 3, 4 e 5, abaixo, , observa-se em média que os pontos experimentais tendem à linha base, pois há a diminuição da atividade Speckle conforme se dá a secagem do material.

Figura 5 - Gráficos experimentais de correlação versus tempo para a cola branca.

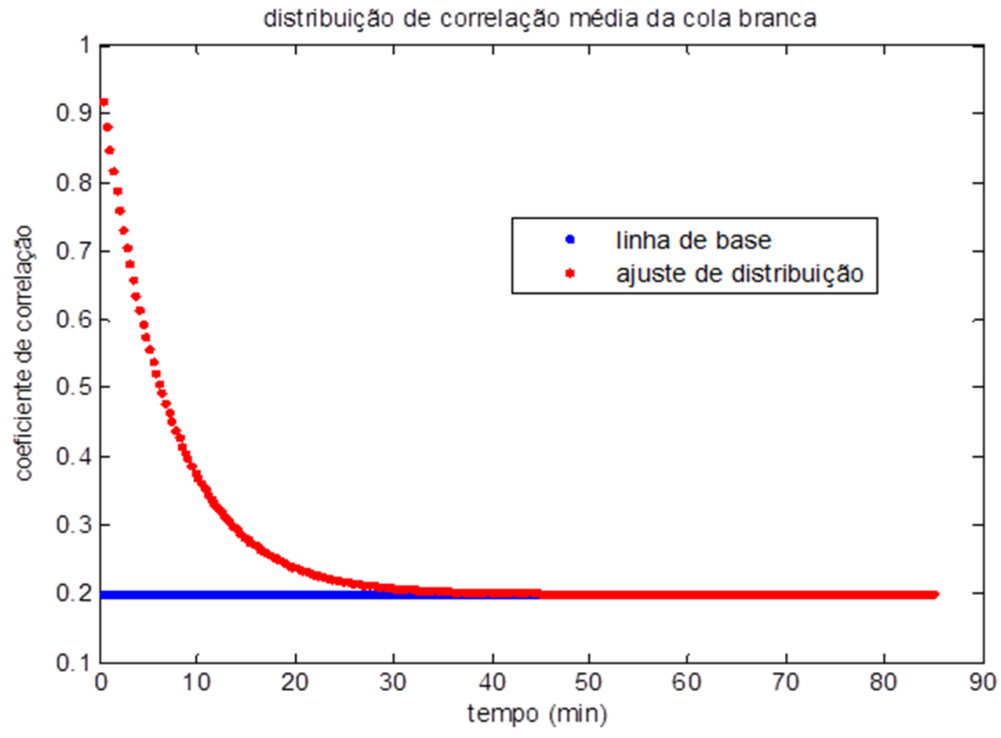

Fonte: Grupo de Óptica e Aplicações da Fatec Itaquera.

Nota-se, também, que os pontos experimentais da amostra de corretivo líquido, por possuir maior velocidade de secagem, aproximam-se muito mais rapidamente dos pontos da linha base em relação às demais amostras.

Segundo a teoria apresentada, um maior nível de atividade Speckle produz uma queda mais acentuada nos coeficientes de correlação. Isso explica o motivo 
das curvas menos acentuadas para as amostras de cola de madeira e cola branca, que apresentam menor nível de atividade Speckle, sendo o menor nível de atividade apresentado pela cola branca.

Com os devidos ajustes dos pontos experimentais, utilizando o método dos mínimos quadrados, foram obtidas as funções correlação $\mu$ versus t, para cada amostra. 0 ponto de intersecção dessas funções com as funções constantes forneram os valores médios dos tempos de secagem das amostras.

A Tabela 2 apresenta os valores dos tempos médios de secagem, das amostras estudadas, acompanhada de seus respectivos erros, obtidos também pelo método dos mínimos quadrados.

Tabela 2 - Valores médios dos tempos de secagem pelo método de correlação.

\begin{tabular}{c|c|cc}
\hline Material & Corretivo líquido & Cola de madeira & Cola tenaz branca \\
\hline Tempo médio de secagem & $(3,76 \pm 0,34) \mathrm{min}$ & $(26,02 \pm 0,44) \mathrm{min}$ & $(37,36 \pm 0,33) \mathrm{min}$ \\
\hline
\end{tabular}

Fonte: Grupo de Óptica e Aplicações da Fatec Itaquera

Como mostra a Tabela 2, o tempo médio de secagem do corretivo líquido é menor em relação aos valores das demais amostras. Isso se explica pelo fato do corretivo líquido ser menos viscoso que as colas e, também, por ser mais volátil ao ambiente estudado em relação às demais amostras. A cola tenaz branca apresentou maior tempo médio de secagem, em relação à cola de madeira, por ser menos volátil.

A Tabela 3 apresenta os resultados obtidos por outro método de análise THSP por Momento de Inércia (MI), apresentado no trabalho de da Silva et al. (2016). Os dados utilizados para análise com MI foram os mesmos utilizados para a análise com o método de correlação, cujos resultados foram apresentados na Tabela 2.

Tabela 3 - Valores médios dos tempos de secagem pelo método de MI.

\begin{tabular}{c|c|cc}
\hline Material & Corretivo líquido & Cola de madeira & Colatenaz branca \\
\hline Tempo médio de secagem & $(3,8 \pm 1,6) \mathrm{min}$ & $(28,0 \pm 4,5) \mathrm{min}$ & $(40,0 \pm 5,0) \mathrm{min}$ \\
\hline
\end{tabular}

Fonte: DA SILVA et al. (2016)

Comparando os resultados das Tabelas 2 e 3 nota-se um aumento significativo da precisão dos tempos de secagem dos valores por correlação em relação aos valores por MI. A incertezado tempo médio de secagem da amostra de corretivo líquido foi da ordemde $9 \%$, no resultado obtido pelo método de correlação, enquanto que para o método de $\mathrm{MI}$ a incerteza foi de $42 \%$.

Para a cola de madeira os valores percentuais são: 1,7\% (correlação) e 16\% (MI). Para a cola tenaz branca os valores percentuais são: 0,9\% (correlação) e 12,5\% (MI). A explicação para essas diferenças está associada aos menores intervalos temporais que foram possíveis de obter com o método de correlação. 


\section{CONCLUSÕES}

0 método apresentado neste trabalho mostrou-se de simples e fácil execução, possibilitando a obtenção de resultados com rapidez e eficiência.

O método de correlação apresentou melhores valores de incerteza em todas as médias, comparados aos valores obtidos na literatura pelo método MI. Isso mostra que para esses tipos de amostras, o método de correlação é mais confiável na obtenção dos tempos de secagem.

Cabe dizer que os resultados obtidos podem ser melhorados se a coleta de dados for realizada com a utilização de lasers de maior coerência temporal e espacial.

Também podem ser adicionados à configuração experimental um filtro espacial e uma lente plano-convexa para transformar a onda gaussiana do laser em onda plana. Finalmente, pode ser acrescentado um sistema de polarizadores para produzir um melhor ajuste de intensidades e uma câmera digital com sensores de melhor qualidade. 


\title{
Study of the drying of materials by means of Dynamic Speckle
}

\begin{abstract}
In this work, Speckle was used to study the average drying time of the liquid corrective, wood glue and white glue samples. Interference patterns were obtained by reflection and captured in a digital camera after the laser interacts with the surfaces of the samples. With the THSP method, the correlation coefficients were obtained and the average drying times of the samples were: $(3,76 \pm 0,34)$ min for the liquid corrective, $(26,02 \pm 0,44)$ min for the wood glue and $(37,36 \pm 0,33)$ min for the white glue.These results were more accurate compared to the values obtained by the moment of inertia method in THSP, found in the literature, showing that the computational method of correlation is more adequate for the set of samples of this work.
\end{abstract}

KEYWORDS: interferometry Speckle; Speckle Correlation; THSP. 


\section{Estudio del secado de materiales por medio de Speckle dinámico}

\section{RESUMEN}

En este trabajo, se utilizó Speckle dinámico para estudiar el tiempo medio de secado en las muestras correctivo líquido, pegamento de madera y cola blanca. Los patrones de interferencia fueron obtenidos por medio de la técnica de Speckle por reflexión y capturados en una cámara digital después de que el láser interagiera con las superficies de las muestras. Con el método THSP se obtuvieron los coeficientes de correlación y por análisis gráficos se encontraron los tiempos medios de secado de las muestras: (3,76 \pm $0,34)$ min para el correctivo líquido, $(26,02 \pm 0,44)$ min para el pegamento de madera y $(37,36 \pm 0,33)$ min para la cola blanca. Estos resultados mostraron mayor precision comparados a los valores obtenidos por método del momento de inércia en THSP, encontrado en la literatura, mostrando que el método computacional de correlaciónesmásadecuado para el conjunto de muestras de ese trabajo.

PALABRAS CLAVE: interferometría Speckle; Correlatión Speckle; THSP. 


\section{Revista Brasileira de Física Tecnológica Aplicada \\ ISSN: 2358-0089}

\section{AGRADECIMENTOS}

Os autores agradecem ao Grupo de óptica e aplicações, GOA, à Câmara de Ensino, Pesquisa e Extensão, CEPE, e à Direção da Fatec Itaquera pelo incentivo e apoio.

\section{REFERÊNCIAS}

ALVES, J. A. Aplicação da técnica do biospeckle laser com abordagem espectral em cenoura minimamente processada. 2012. Dissertação (M estrado em Ciências dos Alimentos) - Universidade Federal de Lavras.

ARFKEN, G.; WEBER, H. H. Física Matemática: métodos matemáticos para engenharia e física. Tradução da 6. Edição por Arlete Simille Marques. Rio de Janeiro: Editora Campus, 2005. 900 p.

ARIZAGA, R.; TRIVI, M.; RABAL, H. Speckle time evolution characterization by the co- occurrence matriz analysis. Optics \& Laser Technology - Elselvier, v.31 p. 163(169), La Plata - Argentina, 1999.

BUTKOV, E. Física Matemática. Tradução de João Bosco Pitombeira Fernandes de Carvalho. 1. Ed. São Paulo: Editora Guanabara, 1988. 725 p.

COSTA, R. M. et al. Técnicas estatísticas aplicadas em imagens do Speckle dinâmico. Rev. Bras. Biom., v. 28, n. 2, p. 27-39, 2010.

DA SILVA, S. L. Estudo de tensões em amostras fotoelásticas com Holografia Digital - Análise quantitativa. 1a Edição: Novas Edições Acadêmicas, 2017, Brasil. $153 \mathrm{p}$.

DA SILVA, S. L. et al.. Estudo do tempo de secagem em amostras líquidas com a técnica de Speckle. Revista Brasileira de Física Tecnológica Aplicada, v. 3, n. 2, p. 30-42, 2016. DOI: 10.3895/rbfta.v3n1.3676.

DA SILVA, S. L. et al. Determinação de propriedades mecânicas do aço 1010 sob tensão no regime elástico por meio de speckle dinâmico. Revista Brasileira de Física Tecnológica Aplicada, v. 4, n. 1, p. 1-15, 2017.

FERREIRA, M . Óptica e Fotónica. Lisboa:Editora Lidel-Edições Técnicas, 2003.

HECHT, E. Óptica. 2 Edição. Lisboa: Fundação Calouste Gulbenkian, 2002. 
HOTTA, V. T.; VIEIRA, M. L. C. Técnicas avançadas em ecocardiografia. 1a Edição: Elsevier, 2015, Brasil. 256 p.

KURAMOTO, R. R. F. Estudo de força eletromotriz de um padrão luminoso oscilante de speckle e suas aplicações. 2011. Dissertação (M estrado em Física) UNICAMP.

M ARCHIORI, M. M.; SOUZA, A. J. Estudo para a proposição de um novo método de medição de rugosidade baseado em reflexão difusa de laser. 70 Congresso Brasileiro de Engenharia de Fabricação, ABCM , 2013.

MIRA, A. R. A Descrição da forma dos grãos de speckle na secagem de adesivo epóxi por expansão em séries de Fourier. 2016. Dissertação (M estrado em Engenharia de Sistemas e Automação) - Universidade Federal de Lavras.

MOREIRA, J. Desenvolvimento de uma ferramenta computacional de livre acesso para o processamento de imagens do speckle laser dinâmico. 2014. Dissertação (Mestrado em Engenharia de Sistemas) - Universidade Federal de Lavras.

NEWTON, I.; ASSIS, A. K. T. Óptica. Tradução da 4. Edição de 1730 por André Koch Torres Assis. São Paulo: EDUSP, 2015. 296 p.

SILVA, E. R.; MURAMATSU, M. O fenômeno do Speckle como introdução à metrologia óptica no laboratório didático, Revista Brasileira de Ensino de Física, v. 29, n. 2, São Paulo, p. 283-286, 2007.

VANIN, V. R.; GOUFFON, P.; HELENE, O. A. M. Tópicos Avançados em Tratamento Estatístico de Dados em Física Experimental. 2. Edição. São Paulo: Eitora Edgard Blucher, 1991. $116 \mathrm{p}$.

Recebido: 08 de agosto de 2017.

Aprovado: 01 de dezembro de 2017

DOI:

Como citar:

DA SILVA, S. L, et al, Estudo da secagem de materiais por meio de Speckle dinâmico. Revista Brasileira

de Física Tecnológica Aplicada, Ponta Grossa, v. 4, n.2, p. 12-25, dezembro de. 2017.

Contato: Sidney Leal da Silva: fatec.professor.sidney@gmail.com

Direito autoral: Este artigo está licenciado sob os termos da Licença Creative Commons-Atribuição 4.0

Internacional.

(c) (1) 